\title{
Acromioclavicular joint instability: anatomy, biomechanics and evaluation
}

\author{
MARISTELLA F. SACCOMANNO, CARMINE DE IESO, GIUSEPPE MILANO
}

Department of Orthopaedics, Catholic University, Division of Orthopaedic Surgery, "A. Gemelli" University Hospital, Rome, Italy

\begin{abstract}
Acromioclavicular (AC) joint instability is a common source of pain and disability. The injury is most commonly a result of a direct impact to the AC joint. The AC joint is surrounded by a capsule and has an intraarticular synovium and an articular cartilage interface. An articular disc is usually present in the joint, but this varies in size and shape. The $\mathrm{AC}$ joint capsule is quite thin, but has considerable ligamentous support; there are four AC ligaments: superior, inferior, anterior and posterior. The coracoclavicular (CC) ligament complex consists of the conoid and trapezoid ligaments. They insert on the posteromedial and anterolateral region of the undersurface of the distal clavicle, respectively. The coracoid origin of the trapezoid covers the posterior half of the coracoid dorsum; the conoid origin is more posterior on the base of the coracoid. Several biomechanical studies showed that horizontal stability of the $\mathrm{AC}$ joint is mediated by the AC ligaments while vertical stability is mediated by the CC ligaments. The radiographic classification of AC joint injuries described by Rockwood includes six types: in type I injuries the AC ligaments are sprained, but the joint is intact; in type II injuries, the AC ligaments are torn, but the CC ligaments are intact; in type III injuries both the $\mathrm{AC}$ and the CC ligaments are torn; type IV injuries are characterized by complete dislocation with posterior displacement of the distal clavicle into or through the fascia of
\end{abstract}

\footnotetext{
Corresponding Author:

Maristella F. Saccomanno, MD

Department of Orthopaedics, Catholic University,

Division of Orthopaedic

"A. Gemelli” University Hospital

Largo A. Gemelli 8, 00168, Rome, Italy

E-mail: maristellasaccomanno@hotmail.it
}

the trapezius; type $\mathrm{V}$ injuries are characterized by a greater degree of soft tissue damage; type VI injuries are inferior $\mathrm{AC}$ joint dislocations into a subacromial or subcoracoid position. The diagnosis of AC joint instability can be based on historical data, physical examination and imaging studies. The cross body adduction stress test has the greatest sensitivity, followed by the AC resisted extension test and the O'Brien test. Proper radiographic evaluation of the $\mathrm{AC}$ joint is necessary. The Zanca view is the most accurate view for examining the AC joint. The axial view of the shoulder is important in differentiating a type III AC joint injury from a type IV injury.

Key Words: acromioclavicular joint, biomechanics, injury, instability, clinical evaluation.

\section{Anatomy}

The acromioclavicular (AC) joint is a frequent site of injuries and degenerative pathology. Physicians must have a good understanding of the anatomy and mechanics of this joint before treating patients with AC joint pathology.

The clavicle has been likened to a crank because of its double curve (1). Its distal, or lateral, end is flattened, and it has prominent landmarks on its inferior surface. The conoid tubercle is located at the most posterior aspect of the clavicle, at the point where the middle third of the shaft curves into the lateral third. The trapezoid ridge extends anteriorly and laterally across the inferior surface of the lateral third of the clavicle. These landmarks represent the insertions of the corresponding ligaments $(2,3)$. The coracoclavicular (CC) ligament complex consists of the conoid and trapezoid ligaments. The former is the postero- 
medial portion, and the latter the anterolateral portion of the CC ligament complex. Bursae can exist between these ligaments. Harris et al. (4) evaluated the anatomical variance of the CC ligaments. The clavicular insertion of the conoid ligament was found to be approximately twice as wide (medial to lateral) and thick (anterior to posterior) as its coracoid insertion, giving rise to its inverted cone shape. The trapezoid ligament was more than three times thicker at its clavicular end than at its coracoid end, but it showed less narrowing of its width compared with the conoid ligament. The coracoid origin of the trapezoid covered the posterior half of the coracoid dorsum; the conoid origin was more posterior on the base of the coracoid, limited anteriorly by the trapezoid insertion. Salter et al. (5) found the trapezoid and conoid ligaments to vary significantly in length and width. The trapezoid ligament varied from $0.8 \mathrm{~cm}$ to $2.5 \mathrm{~cm}$ both in length and in width, while the conoid ligament varied from $0.7 \mathrm{~cm}$ to $2.5 \mathrm{~cm}$ in length and from $0.4 \mathrm{~cm}$ to $0.95 \mathrm{~cm}$ in width. Several studies (69) have shown the center of the trapezoid and the conoid ligament insertion to be located $2.5 \mathrm{~cm}$ and $4.6 \mathrm{~cm}$ from the lateral edge of the clavicle, respectively (Fig. 1). The lateral edges of the trapezoid and the conoid ligament origins were located $11.8 \mathrm{~mm}$ and $25.3 \mathrm{~mm}$ from the distal end of the clavicle, respectively.

The distal clavicle articulates with the acromion via the medial facet, which is oriented posteriorly and laterally; the articular surface of the acromion is directed medially and anteriorly $(3,10)$. The average size of the

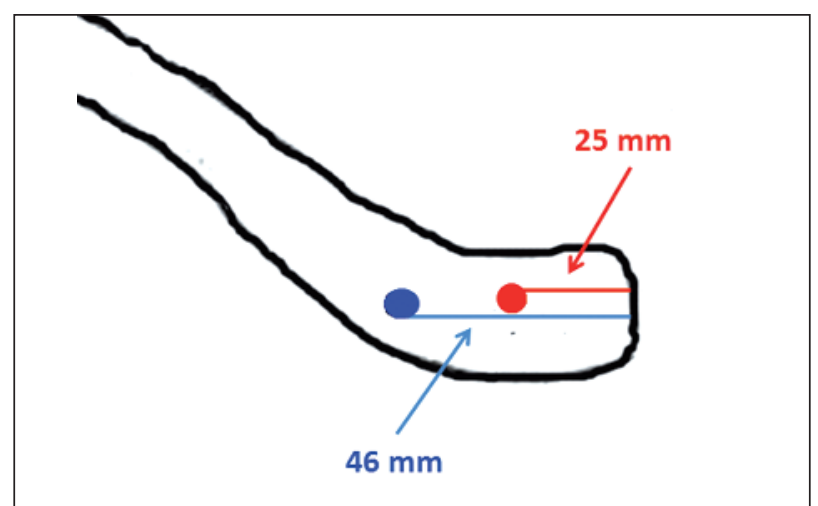

Fig. 1. Clavicular insertions of conoid (blu circle) and trapezoid (red circle) ligaments. joint surfaces in the adult AC joint has been found to be $9 \mathrm{~mm}$ (vertical) by $19 \mathrm{~mm}$ (anteroposterior) (3, 11). The average width of the $\mathrm{AC}$ joint ranges from 1 $\mathrm{mm}$ to $3 \mathrm{~mm}$, and decreases with advancing age, regardless of gender $(12,13)$.

The AC joint is a diarthrodial joint: it is surrounded by a capsule and has an intra-articular synovium and an articular cartilage interface. The hyaline articular cartilage becomes fibrocartilage on the acromial side of the joint by the age of 17 and on the clavicular side by the age of $24(14,15)$.

The AC joint capsule is quite thin, but has considerable ligamentous support; there are four AC ligaments: superior, inferior, anterior and posterior. Salter et al. (5), in a cadaveric study, found that the superior AC ligament was more substantial and thicker (2.0 $\mathrm{mm}-5.5 \mathrm{~mm}$ ) than the inferior AC ligament, and had a more defined insertion into the distal clavicle. Stine et al. (16) reported that the AC joint capsular insertion on the acromion begins, on average, $2.8 \mathrm{~mm}$ (range, 2.3 to $3.3 \mathrm{~mm}$ ) from the medial acromion and begins on the lateral clavicle a mean of $3.5 \mathrm{~mm}$ (range, 2.9 to $3.9 \mathrm{~mm}$ ) from the distal clavicle. The mean capsular width ranged from 1.6 to $2.9 \mathrm{~mm}$. The mean distance from the medial acromion to the AC ligament insertion was $3.5 \mathrm{~mm}$.

An articular disc is usually present in the joint, but this varies in size and shape $(3,10)$. In anatomical studies, De Palma (17) and Salter et al. (5) observed two types of disk: a complete disk (very rare) and a meniscoidlike disk. Recently, Heers et al. (18), in a cadaveric model, identified a superior and inferior wedgeshaped meniscoid disk consisting of fibrocartilaginous tissue. The thickness of this disk varied, ranging from 1.5 to $4 \mathrm{~mm}$. Beginning in the second decade of life, this structure undergoes rapid degeneration; by the fourth decade this degeneration is significant.

The deltoid, trapezius and pectoralis major also have important attachments to the clavicle. The deltoid inserts into the anterior surface of the lateral third of the clavicle, and the trapezius inserts into its posterior aspect. The pectoralis major inserts into the anterior surface of the medial two thirds (2). The acromion has a roughened surface at its anterior tip where the coracoacromial ligament inserts, after giving off fibers that blend medially with the inferior AC ligament $(3,5,19)$. 


\section{Biomechanics}

Awareness of the functional importance of the ligaments surrounding the distal clavicle and $\mathrm{AC}$ joint has grown over time.

Several biomechanical studies (20-30) involving sequential ligament sectioning in cadaveric specimens have documented the strength, stiffness, and relative contribution of these supporting structures to joint constraint under displacement. Fukuda et al. (20) found that the conoid and trapezoid ligaments resisted 50 and $65 \%$ of the forces applied, respectively. At larger displacements, though, the conoid and trapezoid ligaments contributed 70 and $60 \%$, respectively. They also found that the $\mathrm{AC}$ ligaments acted as a primary restraint to posterior displacement of the clavicle and posterior axial rotation, regardless of the degree of displacement. Debski et al. (21) confirmed this latter observation, noting a $100 \%$ displacement in both anterior and posterior directions after transecting the $\mathrm{AC}$ joint capsule. Transection of the capsule resulted in a significant increase $(>200 \%)$ in the mean in situ force in the conoid ligament (to $49 \pm 23 \mathrm{~N}$ ). The mean force in the conoid ligament was also significantly greater than that in the trapezoid $(25 \pm 19 \mathrm{~N})$ after transection of the capsule. The mean in situ force in the trapezoid increased significantly from $23 \pm 15 \mathrm{~N}$ to $38 \pm 23 \mathrm{~N}$, or $66 \%$, in response to posterior loading. The resultant force in the trapezoid was also found to be significantly (approximately 50\%) greater than that in the conoid $(24 \pm 22 \mathrm{~N})$, which had increased only $9 \%$. Thus, conoid and trapezoid function differently in resisting applied loads, depending on the direction of the applied load. After transection of the capsule, the conoid served as the primary restraint against anterior and superior loading, while the trapezoid functioned as the primary restraint against posterior loading. The relative orientation of these two ligaments has been suggested to account for their different functions (20-23). Dawson et al. (24) showed that the AC ligaments offer three times more stability in the anteroposterior plane than in the supero-inferior plane.

This finding agrees with the generally accepted principle that horizontal stability is mediated by the AC ligaments while vertical stability is mediated by the CC ligaments.
Several studies (25-28) evaluating the structural properties of the CC ligaments found no significant differences between them.

Load-to-failure test results confirmed that the conoid ligament fails first when a load is applied to the CC complex in a superior direction $(28,29)$. The most common site of rupture was the midsubstance region, followed by rupture about the origin for all three ligaments $(3,30)$. Isolated, or pure, ruptures from the insertion of origin were unusual and, when seen, were usually "mixed" ruptures, i.e. associated with midsubstance rupture (3).

Recently, Sahara et al. (31), in an in vivo study, analyzed the 3D kinematics of the AC joint during arm abduction using 3D MR images obtained during vertically open MRI. They found that in the anteroposterior direction, the clavicle translated most posteriorly $(-1.9 \pm 1.3 \mathrm{~mm})$ at $90^{\circ}$ of abduction and most anteriorly $(1.6 \pm 2.7 \mathrm{~mm})$ at maximum abduction. This may be due to the influence, on translation, of the muscles around the $\mathrm{AC}$ joint. The anterior fibers of the deltoid muscle and the superior trapezius muscle attach to the anterior and posterior aspect of the distal end of the clavicle, respectively. At $90^{\circ}$ of abduction, the humeral insertion of the deltoid muscle was located in the lateral side of the clavicle, and the traction force of this muscle was directed laterally. Thus the anterior component of the traction force of this muscle may have become smaller than the posterior component of the traction force of the superior trapezius, causing the clavicle to translate posteriorly. At maximum abduction, the humeral deltoid insertion was located in the anterolateral side of the clavicle. Thus the anterior component of the traction force of this muscle may have become larger than the posterior component of the superior trapezius, causing the clavicle to translate anteriorly.

\section{Evaluation}

Acromioclavicular joint instability is a common source of pain and disability, and it occurs both in athletes and in inactive patients. The diagnosis of AC joint instability can be based on historical data, physical examination and imaging studies. 


\section{Toints}

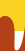
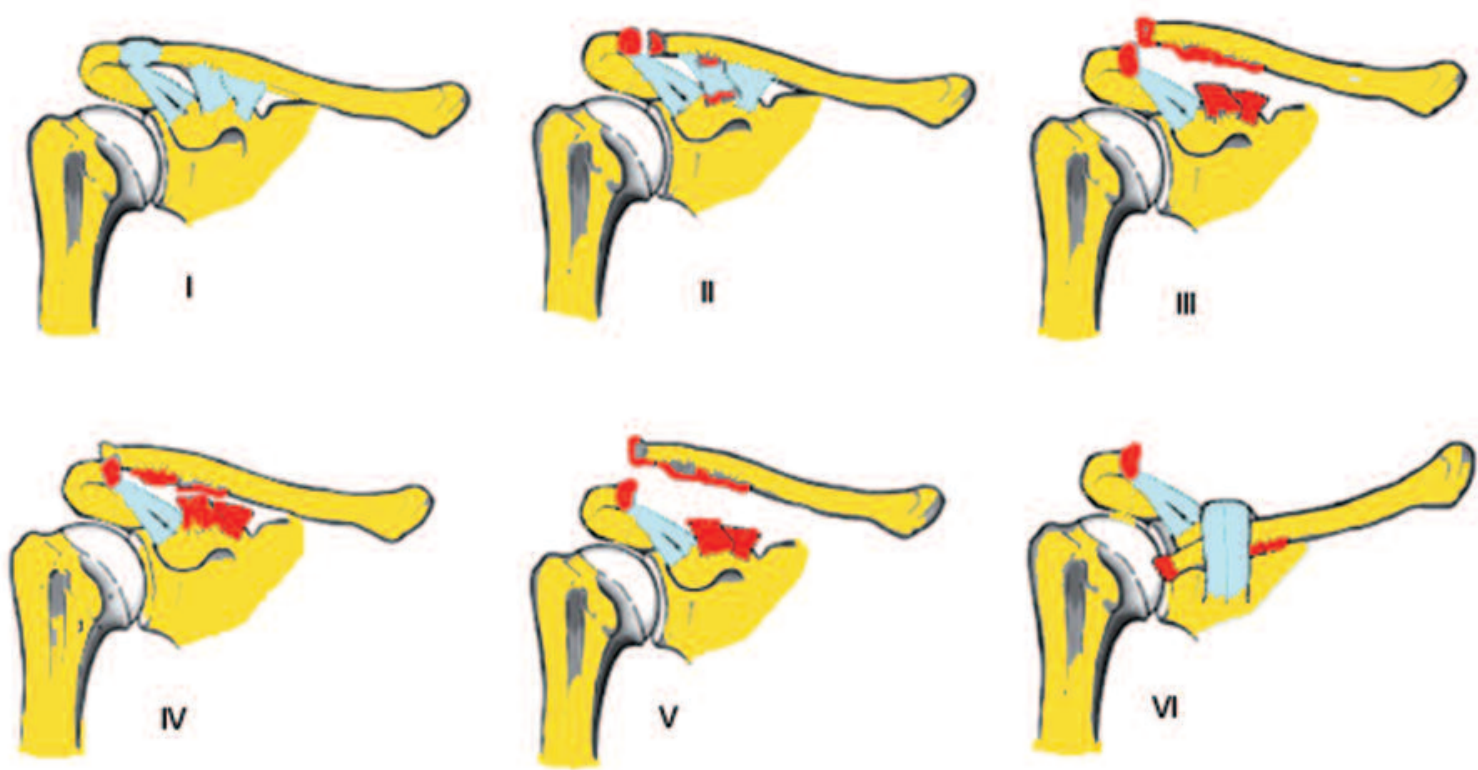

Fig. 2. Classification of AC joint injuries according to Rockwood et al. (14).

Two common mechanisms account for AC joint injury: direct and indirect. The injury is most commonly a result of a direct impact to the AC joint. A direct injury occurs when a person falls onto the AC joint with their arm at their side in an adducted position, as is commonly seen in collision sports such as hockey, football, rugby and karate. An indirect injury to the AC joint can also occur as the result of a fall on an outstretched hand. The fall typically drives the humeral head superiorly into the acromion (15).

The radiographic classification of AC joint injuries, as described by Rockwood et al. (14), represents a continuum of increasingly severe soft tissue injury (Fig. 2). In type I the AC ligaments are sprained, but the joint is intact. Radiographically, there is no widening, separation, or deformity at the AC joint. With type II injuries, the AC ligaments are torn, but the CC ligaments are intact. A bilateral X-ray may demonstrate that the distal clavicle is slightly elevated, but the CC interspace is the same in both the injured and the uninjured shoulder. In type III injuries both the AC and the CC ligaments are torn, but the deltoid and trapezial fascia are intact. X-rays reveal that the distal clavicle is $100 \%$ displaced superiorly in relation to the acromion. Type IV injuries are characterised by complete dislocation with posterior displacement of the distal clavicle into or through the fascia of the trapezius. Type $\mathrm{V}$ injuries are characterised by a greater degree of soft tissue damage, with the deltotrapezial fascia stripped off the acromion and the clavicle. On bilateral X-ray there is a 100-300\% increase in the CC interspace. Type VI injuries are inferior AC joint dislocations into a subacromial or subcoracoid position.

The patient with AC lesions typically complains of pain on the top of the shoulder near the AC joint. The distribution of pain in the presence of pathological lesions of the $\mathrm{AC}$ joint can be into the trapezius or anterior shoulder $(32,33)$.

On physical examination, the patient with AC joint pathological lesions may have swelling or deformity and may have tenderness locally at the AC joint. Several other physical examination signs have been described as provocative for AC joint abnormalities. Chronopoulos et al. (33) found that the cross body adduction stress test showed the greatest sensitivity (77\%), followed by the AC resisted extension test $(72 \%)$ and the O'Brien test (41\%). A shrug test has been described to differentiate a type III injury from a type $\mathrm{V}$ injury. If the joint reduces when the patient shrugs his or her shoulders, then the deltotrapezial fascia is intact and a type $\mathrm{V}$ injury can be ruled out.

When the history and physical examination indicate 
possible AC joint injury, imaging studies are necessary. Proper radiographic evaluation of the $\mathrm{AC}$ joint requires $1 / 3$ to $1 / 2$ of the $\mathrm{X}$-ray penetration needed for glenohumeral joint exposure. Specific directions must be given to the radiology technician in order to obtain the appropriate view. Anteroposterior, lateral and axial views are standard views taken for the shoulder; however, a Zanca view is the most accurate view for examining the AC joint (Fig. 3). This view is performed by tilting the X-ray beam $10^{\circ}$ to $15^{\circ}$ towards the cephalic direction and using only $50 \%$ of the standard shoulder anteroposterior penetration strength.

The axial view of the shoulder is important in differentiating a type III AC joint injury from a type IV injury. Visualization of the scapula anterior to the clavicle will indicate a type IV lesion. When there is a normal CC interspace but a complete dislocation of the AC joint, a coracoid fracture should be suspected. A Stryker notch view is helpful for diagnosing this condition (34). Tauber et al. (35) recently proposed supine dynamic axillary lateral shoulder views, in order to detect horizontal instability of the distal clavicle in patients with acute AC joint dislocations.

The configuration of the AC joint on anteroposterior radiographs varies significantly. Zanca (36) reported that the AC joint width is normally between 1 and 3 $\mathrm{mm}$. Petersson (37) reported that the AC joint space diminishes with increasing age, thus a joint space of 0.5 $\mathrm{mm}$ is normal in 60-year-old patients. The CC interspace can also exhibit variability. Bosworth (11) stated that the average distance between the clavicle and coracoid process is usually between 1.1 and $1.3 \mathrm{~cm}$.

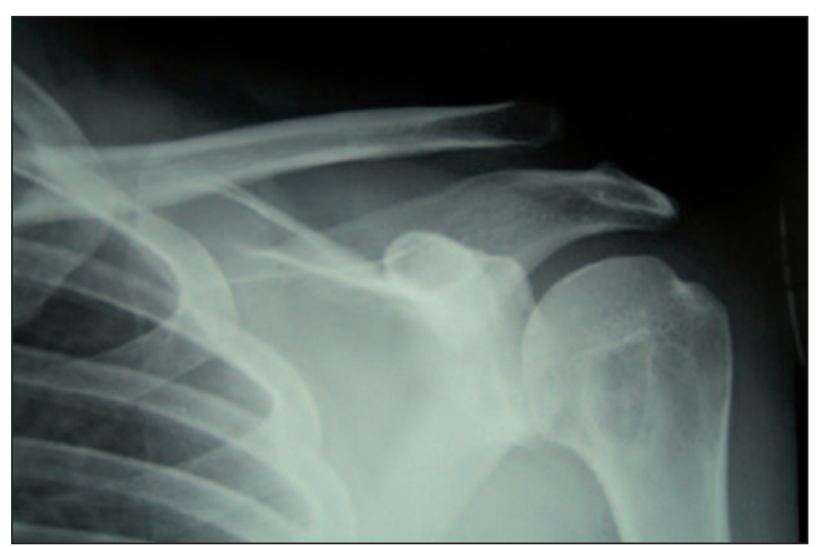

Fig. 3. Zanca view showing a type III AC joint injury.
Although the role of ultrasound in imaging AC joint injuries remains unclear, a recent study (38) using this modality identified abnormal movements of the injured AC joint with cross-arm adduction which were not identified with plain resting or stress radiographs (39). MRI allows excellent anatomical display of AC joint structures and can give clinically relevant information on the type and extension of AC joint trauma, which may influence treatment; however it not feasible for use on a routine basis because of its costs and limited local availability (40).

\section{References}

1. Pratt NE. Anatomy and biomechanics of the shoulder. J Hand Ther. 1994;7:65-76.

2. Klassen J, Morrey BF, Ann KN. Surgical anatomy and the function of the acromioclavicular and coracoclavicular ligaments. Oper Tech Sports Med. 1997;5:60- 64.

3. Renfree KJ, Wright TW. Anatomy and biomechanics of the acromioclavicular and sternoclavicular joints. Clin Sports Med. 2003;22:219-237.

4. Harris RI, Vu DH, Sonnabend DH, et al. Anatomic variance of the coracoclavicular ligaments. J Shoulder Elbow Surg. 2001; 10:585-588.

5. Salter EG Jr, Nasca RJ, Shelley BS. Anatomical observations on the acromioclavicular joint in supporting ligaments. Am J Sports Med. 1987;15:199-206.

6. Boehm TD, Kirschner S, Fischer A, et al. The relation of the coracoclavicular ligament insertion to the acromioclavicular joint. A cadaver study of relevance to lateral clavicle resection. Acta Orthop Scand. 2003;74:718-721.

7. Renfree KJ, Riley MK, Wheeler D, et al. Ligamentous anatomy of the distal clavicle. J Shoulder Elbow Surg. 2003;12:355-359.

8. Rios CG, Arciero RA, Mazzocca AD. Anatomy of the clavicle and coracoid process for reconstruction of the coracoclavicular ligaments. Am J Sports Med. 2007;35:811-817.

9. Salzmann GM, Paul J, Sandmann GH, et al. The coracoidal insertion of the coracoclavicular ligaments: an anatomic study. Am J Sports Med. 2008;36:2392-2397.

10. Tillmann B, Peterson W. Clinical anatomy. In: Fu FH, Ticker JB, Imhoff AB (Eds), Analysis of Shoulder Surgery. Appleton and Lange, Stanford. 1998; 1-29.

11. Bosworth BM. Complete acromioclavicular dislocation. N Engl J Med. 1949;241:221-225.

12. Bonsell S, Pearsell AW 4th, Heitman RJ, et al. The relationship of age, gender, and degenerative changes observed on radiographs of the shoulder in asymptomatic individuals. J Bone Joint Surg Br. 2000;82:1135-1139.

13. Oppenheimer A. Arthritis of the acromioclavicular joint. J Bone Joint Surg. 1943;25: 867-870.

14. Rockwood CA Jr, Williams GR, Young D. Disorders of the acromioclavicular joint. In: Rockwood CA Jr, Matsen F (Eds), The Shoulder. Saunders, Philadelphia. 1998;483-553.

15. Bontempo NA, Mazzocca AD. Biomechanics and treatment of acromioclavicular and sternoclavicular joint injuries. Br J Sports Med. 2010;44:361-369. 


\section{oints}

$\sqrt{2}$

16. Stine IA, Vangsness CT Jr. Analysis of the capsule and ligament insertions about the acromioclavicular joint: a cadaveric study. Arthroscopy. 2009;25:968-974.

17. De Palma AF. Surgical anatomy of the acromioclavicular and sternoclavicular joints. Surg Clin North Am. 1963;43:15411550.

18. Heers G, Götz J, Schubert T, et al. MR imaging of the intraarticular disk of the acromioclavicular joint: a comparison with anatomical, histological and in-vivo findings. Skeletal Radiol. 2007;36:23-28.

19. Shaffer BS. Painful conditions of the acromioclavicular joint. J Am Acad Orthop Surg. 1999;7: 176-188.

20. Fukuda K, Craig EV, An KN, et al. Biomechanical study of the ligamentous system of the acromioclavicular joint. J Bone Joint Surg Am. 1986;68:434-440.

21. Debski RE, Parsons IM 4th, Woo SL, et al. Effect of capsular injury on acromioclavicular joint mechanics. J Bone Joint Surg Am. 2001;83-A:1344-1351.

22. Debski RE, Parsons IM 3rd, Fenwick J, et al. Ligament mechanics during three degree-of-freedom motion at the acromioclavicular joint. Ann Biomed Eng. 2000;28:612-618.

23. Yoo YS, Tsai AG, Ranawat AS, et al. A biomechanical analysis of the native coracoclavicular ligaments and their influence on a new reconstruction using a coracoid tunnel and free tendon graft. Arthroscopy. 2010;26:1153-1161.

24. Dawson PA, Adamson GJ, Pink MM, et al. Relative contribution of acromioclavicular joint capsule and coracoclavicular ligaments to acromioclavicular stability. J Shoulder Elbow Surg. 2009; 18:237-244.

25. Lee KW, Debski RE, Chen $\mathrm{CH}$, et al. Functional evaluation of the ligaments at the acromioclavicular joint during anteroposterior and superoinferior translation. Am J Sports Med. 1997;25:858-862.

26. Harris RI, Wallace AL, Harper GD, et al. Structural properties of the intact and the reconstructed coracoclavicular ligament complex. Am J Sports Med. 2000;28:103-108.

27. Motamedi AR, Blevins FT, Willis MC, et al. Biomechanics of the coracoclavicular ligament complex and augmentations used in its repair and reconstruction. Am J Sports Med. 2000;28: 380-384.
28. Costic RS, Vangura A Jr, Fenwick JA, et al. Viscoelastic behavior and structural properties of the coracoclavicular ligaments. Scand J Med Sci Sports. 2003;13:305-310.

29. Mazzocca AD, Spang JT, Rodriguez RR, et al. Biomechanical and radiographic analysis of partial coracoclavicular ligament injuries. Am J Sports Med. 2008;36:1397-1402.

30. Costic RS, Labriola JE, Rodosky MW, Debski RE. Biomechanical rationale for development of anatomical reconstructions of coracoclavicular ligaments after complete acromioclavicular joint dislocations. Am J Sports Med. 2004;32:1929-1936.

31. Sahara W, Sugamoto K, Murai M, et al. 3D kinematic analysis of the acromioclavicular joint during arm abduction using vertically open MRI. J Orthop Res. 2006;24:1823-1831.

32. Gerber C, Galantay RV, Hersche O. The pattern of pain produced by irritation of the acromioclavicular joint and the subacromial space. J Shoulder Elbow Surg. 1998;7:352-355.

33. Chronopoulos E, Kim TK, Park HB, et al. Diagnostic value of physical tests for isolated chronic acromioclavicular lesions. Am J Sports Med. 2004;32:655-661.

34. Mazzocca AD, Arciero RA, Bicos J. Evaluation and treatment of acromioclavicular joint injuries. Am J Sports Med. 2007;35:316-329.

35. Tauber M, Koller H, Hitzl W, et al. Dynamic radiologic evaluation of horizontal instability in acute acromioclavicular joint dislocations. Am J Sports Med. 2010;38:1188-1195.

36. Zanca P. Shoulder pain: involvement of the acromioclavicular joint. (Analysis of 1,000 cases). Am J Roentgenol Radium Ther Nucl Med. 1971;112:493-506.

37. Petersson C. Degeneration of the acromioclavicular joint. A morphological study. Acta Orthop Scand. 1983;54:434-438.

38. Peetrons P, Bédard JP. Acromioclavicular joint injury: enhanced technique of examination with dynamic maneuver. J Clin Ultrasound. 2007;35:262-267.

39. Rios CG, Mazzocca AD. Acromioclavicular joint problems in athletes and new methods of management. Clin Sports Med. 2008;27:763-788.

40. Schaefer FK, Schaefer PJ, Brossmann J, Hilgert RE, Heller $\mathrm{M}$, Jahnke T. Experimental and clinical evaluation of acromioclavicular joint structures with new scan orientations in MRI. Eur Radiol. 2006;16:1488-1493. 